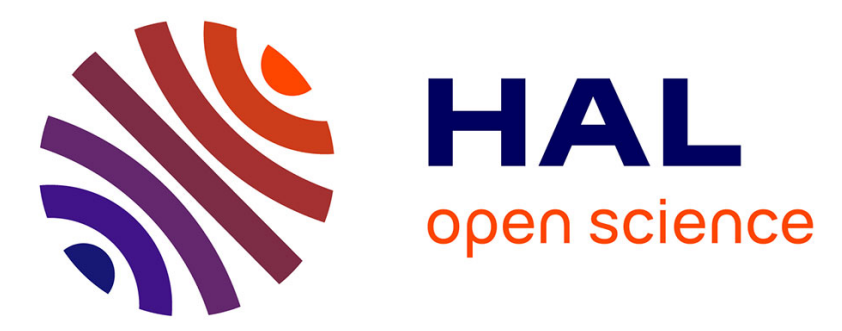

\title{
Diffraction symmetry of binary Fourier elements with feature sizes on the order of the illumination wavelength and effects of fabrication errors
}

\author{
Giang Nam Nguyen, Kevin Heggarty, Julien Le Meur, Andreas Bacher, \\ Patrick Meyrueis
}

\section{To cite this version:}

Giang Nam Nguyen, Kevin Heggarty, Julien Le Meur, Andreas Bacher, Patrick Meyrueis. Diffraction symmetry of binary Fourier elements with feature sizes on the order of the illumination wavelength and effects of fabrication errors. Optics Letters, 2017, 42 (24), pp.5178 - 5181. 10.1364/OL.42.005178 . hal-01812647

\section{HAL Id: hal-01812647 \\ https://hal.science/hal-01812647}

Submitted on 12 Jul 2018

HAL is a multi-disciplinary open access archive for the deposit and dissemination of scientific research documents, whether they are published or not. The documents may come from teaching and research institutions in France or abroad, or from public or private research centers.
L'archive ouverte pluridisciplinaire HAL, est destinée au dépôt et à la diffusion de documents scientifiques de niveau recherche, publiés ou non, émanant des établissements d'enseignement et de recherche français ou étrangers, des laboratoires publics ou privés. 


\title{
Diffraction symmetry of binary Fourier elements with feature sizes on the order of the illumination wavelength and effects of fabrication errors
}

\author{
Giang-Nam Nguyen, ${ }^{1,4, *}$ Kevin Heggarty, ${ }^{1}$ Julien Le Meur, ${ }^{1}$ Andreas Bacher, ${ }^{2}$ and Patrick Meyrueis ${ }^{3}$ \\ 'Optics Department, Telecom Bretagne, Avenue du Technopole 655, 29238 Brest, France \\ ${ }^{2}$ Institute for Microstructure Technology, Karlsruhe Institute of Technology, 76344 Karlsruhe, Germany \\ ${ }^{3}$ Icube Laboratory, University of Strasbourg, Pole API, 300 Boulevard S. Brant, 67412 Illkirch Cedex, France \\ ${ }^{4}$ Currently at Visteon $\mathrm{GmbH}$, An der RaumFabrik 33b, 76227 Karlsruhe, Germany \\ *Corresponding author: gnguyen1@visteon.com
}

\begin{abstract}
When building spot array binary Fourier diffractive optical elements (DOEs) having feature sizes on the order of the wavelength, we noticed remarkable variations in the experimental diffraction efficiency compared to the simulation results. Even with the use of high-cost electron beam lithography and rigorous Fourier modal method simulations, there appear to be no publications, to the best of our knowledge, showing close agreement in diffraction efficiency between the simulation and experimental results. In this Letter, we show that the diffraction symmetry of binary Fourier DOEs can be an efficient and consistent metric for evaluating the limit of the thin-element approximation and the effects of fabrication errors. of America
\end{abstract}

OCIS codes: (090.1970) Diffractive optics; (220.0220) Optical design and fabrication; (220.3740) Lithography; (050.6624) Subwavelength structures; (220.4241) Nanostructure fabrication.

https://doi.org/10.1364/OL.42.005178

As diffractive optical element (DOE) fabrication techniques have improved, it has become common to fabricate DOEs with feature sizes close to the limits of the scalar diffraction models generally used to design DOEs. More rigorous vector models are available, but their computational complexity often limits their application to relatively simple DOEs (e.g., $1 \mathrm{D}$, periodic, small extent). Thus, there is an increasingly important need for ways to determine when designing a DOE whether scalar models are sufficiently accurate or whether vector models are necessary. Unfortunately, there appears to be no general agreement in the literature on this question. Several authors [1] and recent publications [2] indicate that since the Rayleigh-Sommerfeld diffraction integral is rigorous for free-space propagation [3], the validity of the scalar diffraction model primarily depends on the thin-element approximation (TEA), which is used to model the DOE region $[4,5]$. Although rigorous vector diffraction models have been used for decades [6,7], there appears to be little or no experimental work verifying the limits of the TEA in practice, as it is often difficult to separate the simulation errors from the fabrication errors [8]. Knowledge of fabrication errors for the zeroth order has been used for precompensation in the design and fabrication process to increase the performance of the fabricated DOEs $[9,10]$. However, the errors in the non-zero orders are less studied and understood. In this Letter, by comparing TEA-based scalar diffraction models with rigorous Fourier modal method (FMM) simulations and the experimental results, the practical limit of the TEA will be examined. We show that the symmetry of the DOE diffraction pattern can be used as a simple, yet efficient, metric for evaluating the accuracy of the TEA.

When building spot array binary Fourier DOEs with feature sizes on the order of the wavelength, we noticed remarkable variations in the symmetry of diffraction efficiency. In this Letter, we will assess the limit of the TEA in predicting two types of symmetries that are frequently used in practice, which are Hermitian and reflection symmetry [5,11]. Figure 1(a) shows a reflection symmetric binary phase Fourier DOE, which

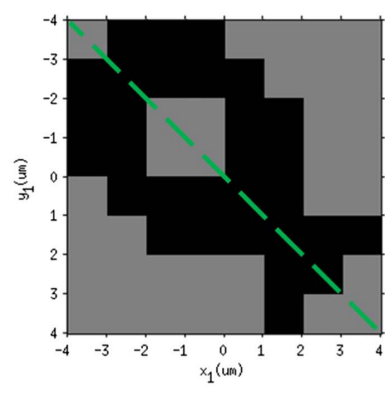

(a)

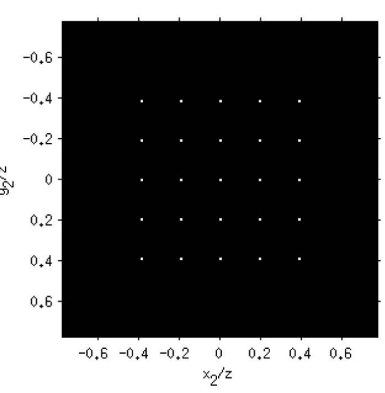

(b)
Fig. 1. (a) Binary Fourier DOE, where the dashed line is the reflection symmetry axis. (b) Target image. 
Table 1. Experimental Diffraction Efficiency $\eta(m, n)(\%)$ Obtained in Diffraction Order $(m, n)$ for the Fabricated DOE

\begin{tabular}{llcccc}
\hline & \multicolumn{5}{c}{$\boldsymbol{n}$} \\
\cline { 2 - 6 } $\boldsymbol{m}$ & $\mathbf{- 2}$ & $\mathbf{- 1}$ & $\mathbf{0}$ & $\mathbf{1}$ & $\mathbf{2}$ \\
\hline 2 & 1.74 & 1.81 & 2.86 & 3.59 & 1.98 \\
1 & 1.77 & 2.50 & 2.86 & 4.20 & 3.07 \\
0 & 2.84 & 2.90 & 3.98 & 2.95 & 2.69 \\
-1 & 3.72 & 4.43 & 3.02 & 1.70 & 2.90 \\
-2 & 2.05 & 3.09 & 2.90 & 2.80 & 1.09 \\
\hline
\end{tabular}

was designed to generate a $5 \times 5$ spot array, as illustrated in Fig. 1(b).

The experimental results of this DOE are shown in Table 1, where the DOE was fabricated at a pixel size of $1 \mu \mathrm{m}$ using electron beam lithography [12], and the DOE illumination wavelength was $633 \mathrm{~nm}$. At this wavelength, the refractive index of the substrate is $n=1.4574$, which means that the etching depth should be about $d=\lambda / 2(n-1) \approx 692 \mathrm{~nm}$ to obtain a $\pi$ phase shift between binary levels [8]. Figure 2 shows the atomic force microscope (AFM) measurement results of a test DOE, with an etching depth of about $700 \mathrm{~nm}$.

As the designed DOE was a binary phase, we expected Hermitian symmetry [5] but, in fact, we observed reflection symmetry in the experimental diffraction pattern. For instance, the spot diffraction efficiency $\eta(1,-2)$ should be the same as $\eta(-1,2)$ but, in fact, they are very different from each other (i.e., $1.77 \%$ and $2.90 \%$, respectively). Instead, $\eta(1,-2)$ and $\eta(2,-1)$ are very similar (i.e., $1.77 \%$ and $1.81 \%$, respectively). Further analysis shows, within experimental errors, a reflection symmetry of the diffraction pattern in the same axis as the reflection symmetry of the designed DOE, which is the dashed line shown in Fig. 1(a).

The far-field diffraction pattern of this DOE with refractive index $n=1.4574$ and feature size $s=1 \mu \mathrm{m}$ was then reconstructed in simulation at $\lambda=633 \mathrm{~nm}$ using the TEA for the DOE region and our scalar non-paraxial propagator for the free-space propagation region [2]. As the rigorous RayleighSommerfeld diffraction integral had been shown to be equivalent to our scalar non-paraxial diffraction model [2], which is based on a single Fourier transform (FT), we would expect the symmetry of the far-field diffraction pattern to depend on the symmetry of the FT. At the optimum etching depth predicted by the TEA, the phase shift between the binary levels is $\pi$,

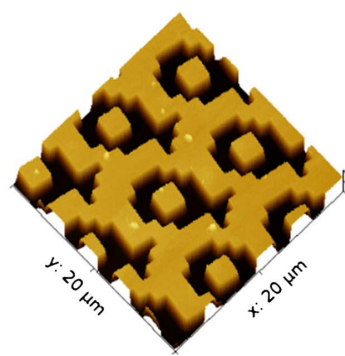

(a)

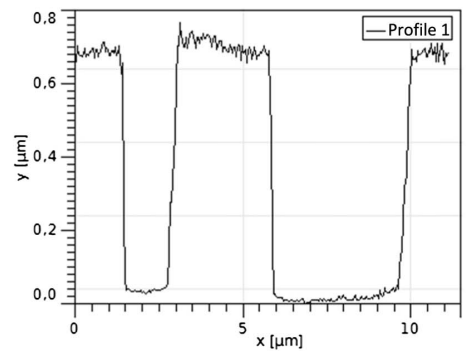

(b)
Fig. 2. (a) AFM image of the DOE. (b) Etching profile of the DOE. which means that the diffracted field after a binary DOE can be represented in the TEA by a real function $f(x, y)$ (i.e., $e^{j \cdot 0}=1, e^{j \cdot \pi}=-1$ ). Therefore, the diffraction pattern $F(X, Y)$ should have Hermitian symmetry [13]:

$$
f(x, y)=f^{*}(x, y) \rightarrow F(X, Y)=F^{*}(-X,-Y),
$$

where $F(X, Y)$ is the FT of $f(x, y)$ and the symbol $*$ denotes the complex conjugate. This means that the diffracted powers of the spots should be Hermitian symmetric, as $|F(X, Y)|^{2}=|F(-X,-Y)|^{2}$. Simulations show that for general binary DOEs (i.e., not at $\pi$ phase shift), scalar theory also leads to Hermitian diffraction symmetry.

Moreover, as the DOE is reflection symmetric, as shown in Fig. 1(a), the scalar theory predicts that, as it is based on a single FT, the far-field diffraction pattern should have the same reflection symmetry [13]:

$$
f(x, y)=f(-y,-x) \rightarrow F(X, Y)=F(-Y,-X) .
$$

Table 2 lists the simulated diffraction efficiency of different diffraction orders $\eta(m, n)$ obtained by the TEA and our scalar model. Both Hermitian and reflection symmetry can clearly be seen, i.e., $\eta(m, n)=\eta(-m,-n)$ and $\eta(m, n)=\eta(-n,-m)$, with $m, n=-2, \ldots, 2$.

For reference, the FMM, which is a rigorous vector diffraction method included in LightTrans VirtualLab [14], was used, where the DOE structure was defined in the programmable interface. Table 3 shows the simulation results for linearly polarized illumination at $45^{\circ}$. Although the predicted diffraction angles are the same as in the scalar simulation, the predicted diffraction efficiencies differ considerably. In particular, the symmetry of the diffracted pattern changes, e.g., $\eta(1,-2)$ and $\eta(-1,2)$ are different from each other (i.e., $2.13 \%$ and $2.91 \%$, respectively). Instead, the diffraction efficiencies $\eta(1,-2)$ and $\eta(2,-1)$ are exactly the same (i.e., $2.13 \%$ ). Further analysis shows that the diffraction pattern is no longer

Table 2. Simulated Diffraction Efficiency $\eta(m, n)(\%)$ of the DOE Using Our Scalar Propagator

\begin{tabular}{llcccc} 
& \multicolumn{5}{c}{$\boldsymbol{n}$} \\
\cline { 2 - 6 } $\boldsymbol{m}$ & $\mathbf{- 2}$ & $\mathbf{- 1}$ & $\mathbf{0}$ & $\mathbf{1}$ & $\mathbf{2}$ \\
\hline 2 & 1.81 & 3.14 & 3.63 & 4.36 & 3.17 \\
1 & 3.14 & 2.45 & 3.18 & 4.26 & 4.36 \\
0 & 3.63 & 3.18 & 0.36 & 3.18 & 3.63 \\
-1 & 4.36 & 4.26 & 3.18 & 2.45 & 3.14 \\
-2 & 3.17 & 4.36 & 3.63 & 3.14 & 1.81 \\
\hline
\end{tabular}

Table 3. Simulated Diffraction Efficiency $\eta(m, n)(\%)$ of the DOE Using FMM for Linearly Polarized Illumination at $45^{\circ}$

\begin{tabular}{llcccc}
\hline & \multicolumn{5}{c}{$\boldsymbol{n}$} \\
\cline { 2 - 6 } $\boldsymbol{m}$ & $\mathbf{- 2}$ & $\mathbf{- 1}$ & $\mathbf{0}$ & $\mathbf{1}$ & $\mathbf{2}$ \\
\hline 2 & 1.70 & 2.13 & 3.08 & 3.85 & 2.18 \\
1 & 2.13 & 2.70 & 3.04 & 4.10 & 3.29 \\
0 & 3.08 & 3.04 & 1.89 & 3.10 & 3.17 \\
-1 & 3.85 & 4.10 & 3.10 & 1.75 & 2.91 \\
-2 & 2.18 & 3.29 & 3.17 & 2.91 & 1.00 \\
\hline
\end{tabular}




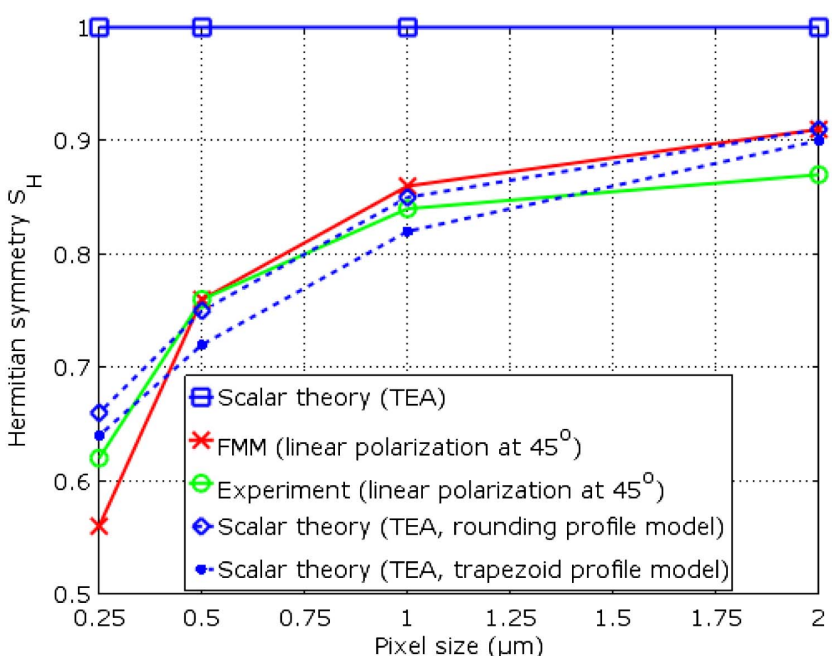

Fig. 3. Average Hermitian symmetry at different DOE feature sizes.

Hermitian symmetric, while the reflection symmetry remains. This is because the phase of the diffracted field after the structure is a continuous function instead of a binary phase shift between binary levels. As the result, the diffracted field after the structure is a complex function, so the far-field diffraction pattern is not Hermitian symmetric.

In order to show the limit of the TEA in predicting the diffraction symmetry of binary Fourier elements, we define an average Hermitian symmetry factor as follows:

$$
S_{H}=\frac{1}{N_{H}} \sum s, \quad \text { where } s=\frac{\eta(m, n)}{\eta(-m,-n)}, \quad \text { with } s \leq 1 .
$$

In this expression, $N_{H}$ is the number of Hermitian symmetric pairs of diffraction spots predicted by the TEA and the freespace propagator, e.g., $N_{H}=12$ for the case of our $5 \times 5$ spot array DOE. The far-field diffraction patterns of the DOE at different feature sizes were reconstructed using the FMM. The average Hermitian symmetry factor was calculated at different feature sizes and shown in Fig. 3. It can be seen that Hermitian symmetry reduces significantly with smaller pixel sizes, in comparison with the scalar prediction $S_{H}=1$. The experimental results show good agreement, within experimental errors, to the FMM simulation.

Similar to the average Hermitian symmetry factor, we define an average reflection symmetry factor of the diffracted spot powers as follows:

$$
S_{R}=\frac{1}{N_{R}} \sum s, \quad \text { where } s=\frac{\eta(m, n)}{\eta(-n,-m)}, \quad \text { with } s \leq 1 .
$$

In this expression, $N_{R}$ is the number of reflection symmetric pairs predicted by the TEA and the free-space propagator, e.g., $N_{R}=10$ for the case of our DOE. Figure 4 shows the average reflection symmetry factor at different feature sizes, where both the scalar theory and FMM simulation with linear polarization at $45^{\circ}$ predict that $S_{R}=1$. The measured reflection symmetry is very close to the theoretical value, except at a $0.25 \mu \mathrm{m}$ pixel size.

Our FMM simulations assume a perfect fabrication with no proximity effect pixel rounding taken into account. When the structure size approaches the limits of the fabrication

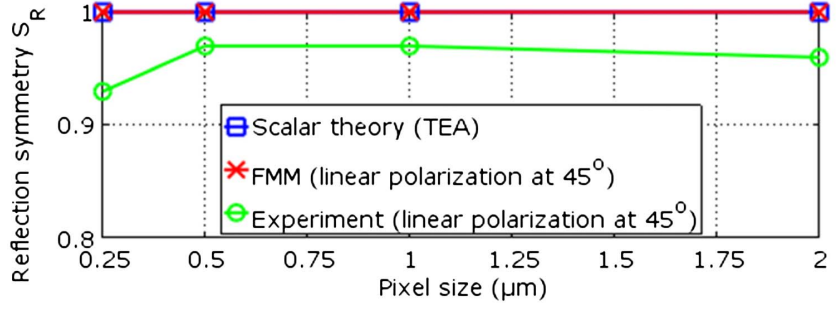

Fig. 4. Average reflection symmetry at different DOE feature sizes.

technology, such rounding effects will start to occur, and deviations between simulated and experimental performance are to be expected. This would appear to explain the increased differences observed between the FMM simulations and the experimental results for the $0.25 \mu \mathrm{m}$ pixel size. Due to fabrication errors, the edge between the phase levels of the fabricated DOE is not perfectly sharp, but rather smooth, as shown in Fig. 2(b). The fabricated DOE becomes, in effect, a multiphase level element; the transmission function is no longer real and, therefore, the diffraction pattern is no longer Hermitian symmetric. If the optical setup of the lithography is perfectly aligned, the fabrication errors will be symmetric, and the fabricated DOE would still retain the symmetry of the designed DOE, as would the experimental diffraction pattern. To investigate this smoothing effect on diffraction efficiency in simulation, it is therefore necessary to oversample the DOEs. We sampled at $125 \mathrm{~nm}$ resolution and applied different smoothing filters [15] to the DOE pattern. These filters correspond to different models for the proximity effect in fabrication, as shown in Fig. 5.

Simulations using our scalar non-paraxial propagator show that the diffraction pattern of the smoothed structure is no longer Hermitian symmetric, but still reflection symmetric. The average Hermitian symmetry of the smoothed structures using $3 \times 3$ filters is added in Fig. 3 for comparison, where they are quite close to the FMM simulation and the experimental result. These results show that the Hermitian symmetry of the diffraction pattern of a binary phase DOE appears to be a useful indicator of the practical limit of the TEA model. If the diffraction pattern maintains Hermitian symmetry, the TEA is valid and can be used to design DOEs. If the diffraction pattern loses Hermitian symmetry, the TEA is no longer valid and more sophisticated models taking into account the electromagnetic fields inside the DOEs structure are necessary. The Hermitian symmetry factor we introduced allows this distinction to be made with a degree of independence from the precise values of the diffraction efficiency of the different diffracted orders and, hence, of the fabrication errors. In terms of reflection symmetry, we see that diffraction symmetry of a binary Fourier DOE can also be used to determine whether the

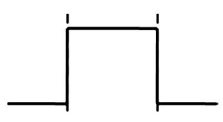

(a)

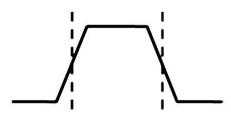

(b)

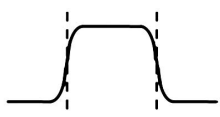

(c)
Fig. 5. Different models for the etching profile: (a) flawless model, (b) trapezoid model, and (c) rounding model. 
lithographic fabrication setup is well aligned, i.e., the DOE structure is still reflection symmetric, despite the proximity effect at the resolution limit of the lithography system.

For general binary phase DOEs without reflection symmetry, the output pattern will always show Hermitian symmetry if the TEA is valid because the DOE transmission is real. The Hermitian symmetry factor introduced here, therefore, can be used to assess the validity of the TEA approximation for all such binary phase DOEs. For DOEs to be used with different polarized illuminations, more detailed investigation using, for example, FMM simulations would be required to assess the polarization effects, but the approach indicated here would still be a useful guide to the closeness to the potential limits of the TEA. Future work should also concentrate on the FMM simulation of the smoothed structure to take into account the effects of fabrication errors on the diffraction efficiency.

In summary, scalar models that make some allowance for the "thickness" of the DOE structure, for example, by modeling fabrication defects as a multi-phase level DOE rather than a binary phase DOE, appear to be a useful intermediate modeling technique enabling the use of computationally expensive full vector models to be postponed. In the example studied here, the limit of the TEA model seems to be when DOE feature sizes are about twice the illuminating wavelength, but further investigation is required on this point, particularly on the relative importance of the structure's width and height.
Acknowledgment. The authors would like to thank Prof. Pierre-Emmanuel Durand for the AFM measurements.

\section{REFERENCES}

1. J. E. Harvey, D. Bogunovic, and A. Krywonos, Appl. Opt. 42, 1167 (2003).

2. G. N. Nguyen, K. Heggarty, P. Gerrard, B. Serio, and P. Meyrueis, Appl. Opt. 53, 2196 (2014).

3. D. G. Voelz, Computational Fourier Optics: A Matlab Tutorial (SPIE, 2011).

4. S. Mellin and G. Nordin, Opt. Express 8, 705 (2001).

5. J. W. Goodman, Introduction to Fourier Optics (McGraw-Hill, 1996).

6. M. G. Moharam, E. B. Grann, D. A. Pommet, and T. K. Gaylord, J. Opt. Soc. Am. A 12, 1068 (1995).

7. M. G. Moharam, D. A. Pommet, E. B. Grann, and T. K. Gaylord, J. Opt. Soc. Am. A 12, 1077 (1995).

8. D. C. O'shea, T. J. Suleski, A. D. Kathman, and D. W. Prather, Diffractive Optics: Design, Fabrication, and Test (SPIE, 2004).

9. D. C. O'shea, Appl. Opt. 34, 6533 (1995).

10. B. C. Kress and P. Meyrueis, Applied Digital Optics: From Microoptics to Nanophotonics (Wiley, 2009).

11. R. L. Morrison, J. Opt. Soc. Am. A 9, 464 (1992).

12. Karlsruhe Nano Micro Facility, 2017, www.knmf.kit.edu.

13. J. F. James, A Student's Guide to Fourier Transforms: With Applications in Physics and Engineering (Cambridge University, 2011).

14. LightTrans GmbH, VirtualLab, 2017, www.lighttrans.com.

15. R. C. Gonzalez and R. E. Woods, Digital Image Processing (PrenticeHall, 2007). 